\title{
Design of a diagnostic residual gas analyzer for the ITER divertor ${ }^{\S}$
}

\author{
C.C. Klepper ${ }^{\mathrm{a}}{ }^{+}$, T.M. Biewer ${ }^{\mathrm{a}}$, V.B. Graves ${ }^{\mathrm{a}}$, P. Andrew ${ }^{\mathrm{b}}$, P.C. Lukens ${ }^{\mathrm{c}}$, C. Marcus ${ }^{\mathrm{a}}$, M. Shimada, ${ }^{\text {b,* }}$, \\ S. Hughes ${ }^{\mathrm{b}}$, B. Boussier ${ }^{\mathrm{b}}$, D.W. Johnson ${ }^{\mathrm{d}}$, W.L. Gardner ${ }^{\mathrm{c}}$, D.L. Hillis ${ }^{\mathrm{a}}$, G. Vayakis $^{\mathrm{b}}$ and M. Walsh $^{\mathrm{b}}$ \\ ${ }^{a}$ Oak Ridge National Laboratory, Oak Ridge, TN 37831-6169, USA \\ ${ }^{b}$ ITER Organisation, Route de Vinon-sur-Verdon, 13067 St. Paul-lez-Durance, France \\ ${ }^{c}$ US ITER Project Office, 1055 Commerce Park Dr \#1, Oak Ridge, TN 37830, USA \\ ${ }^{d}$ US ITER Diagnostics Office, Princeton Plasma Physics Laboratory, Princeton, NJ, 08540, USA
}

\begin{abstract}
One of the ITER diagnostics having reached an advanced design stage is a diagnostic RGA for the divertor, i.e. residual gas analysis system for the ITER divertor, which is intended to sample the divertor pumping duct region during the plasma pulse and to have a response time compatible with plasma particle and impurity lifetimes in the divertor region. Main emphasis is placed on helium (He) concentration in the ducts, as well as the relative concentration between the hydrogen isotopes (mainly in the form of diatomic molecules of $\mathrm{H}, \mathrm{D}$, and T). Measurement of the concentration of radiative gases, such as neon $(\mathrm{Ne})$ and nitrogen $\left(\mathrm{N}_{2}\right)$, is also intended. Numerical modeling of the gas flow from the sampled region to the cluster of analysis sensors, through a long ( $\sim 8 \mathrm{~m}$ long, $\sim 110 \mathrm{~mm}$ diameter) sampling pipe originating from a pressure reducing orifice, confirm that the desired response time $\left(\sim 1 \mathrm{~s}\right.$ for $\mathrm{He}$ or $\left.\mathrm{D}_{2}\right)$ is achieved with the present design.
\end{abstract}

Keywords: ITER, Fusion Energy, Residual Gas Analysis, Plasma Diagnostic

\section{Introduction}

The US-ITER Domestic Agency project to develop the ITER Diagnostic Residual Gas Analyzer (DRGA) is currently entering its Final Design stage. The base design includes two, independent, complete systems, each integrating three measurement sensors to analyze the neutral gas composition in the main chamber and in the divertor duct. Main emphasis is placed on $\mathrm{He}$ concentration in the ducts, as well as the relative concentration between the hydrogen isotopes. Measurement of the concentration of radiative gases, such as $\mathrm{Ne}$ and $\mathrm{N}_{2}$, is also intended. More recently, the detection of potentially forming (deuterated, tritiated) ammonia has become of interest and is being explored.

As part of ITER's plasma diagnostics set, the DRGA is designed to operate throughout the plasma discharge, which will have a pulse length as long as 3000 s, and with sufficient response time to resolve gas concentrations in scale times compatible with plasmawall interactions $(\sim 1 \mathrm{~s}$ for the divertor system somewhat higher for the non- hydrogenic species and 10x higher for the main chamber). To achieve these capabilities, these systems are being engineered with physics sensors compatible with the harsh environment of the ITER port-cell, using special, radiation hardened sensors where possible and with most electronics separated from the sensors and radiation shielded. With working gas pressures as high as $10 \mathrm{~Pa}$ anticipated for the ITER divertor ducts, the divertor DRGA system is designed to maintain pressure differences of 3-4 orders of magnitude across its chamber. The positioning divertor system's analysis chamber in a port-cell (an area on the shielded side of the biological shield and accessible during maintenance periods) has required a nearly $8 \mathrm{~m}$ long extension of ITER's primary vacuum. Traversing the toroidal field cryostat and terminating inside the pumping duct region of the primary vacuum chamber, with a pressure-reducing orifice, this vacuum extension serves as the divertor DRGA's sampling pipe. In spite of this substantial separation between the measurement and the sampled region, gas flow calculations show that the $\sim 1 \mathrm{~s}$ response time is maintained for the divertor DRGA. The capability of the present design to meet the response time requirement, as well as the physics significance of this measurement requirement, is central to this paper. It is important, since it is the relevance to processes and associates timescales in the plasma boundary that differentiates a diagnostic RGA from a vacuum integrity monitoring RGA.

The present paper primarily focuses on the requirements and present design of the divertor DRGA, which is presently more evolved that the main chamber DRGA. The requirements driving the design are discussed in Section 2, and the design to meet these requirements is described in Section 3.

\section{Requirements for the ITER divertor DRGA}

\subsection{Response time}

The ITER diagnostic requirements [Ref 1, Donne '07] specify a 1s nominal response time for the gas composition in the divertor ducts. To better understand the significance of, as well as the tolerance for, this specification, it is best to consider the particle balance time scales anticipated for the ITER plasma boundary.

\footnotetext{
§Work supported, in part, by the US DOE under Contract No. DE-AC05-000R22725 with UT-Battelle, LLC. The views and opinions expressed herein do not necessarily reflect those of the ITER Organization

tkleppercc@ornl.gov

*Presently with the International Fusion Energy Research Centre, Rokkasho-mura, Kamikita-gun, Aomori 039-3212, shimada.michiya@jaea.go.jp
} 
For ITER, the deuterium-tritium (DT) gas fuelling rate will be in the range $30-200 \mathrm{~Pa}-\mathrm{m}^{3} / \mathrm{s}$, or $0.16-$ $1.1 \times 10^{23}$ particles/s. The DT particles in the core plasma, given known volume and anticipated plasma density profile, is estimated at $1 \times 10^{23}$ particles. The ratio of these two quantities gives the global particle replacing time in the range of $1-6 \mathrm{~s}$. This is the main scientific reason for seeking a response time as low as $1 \mathrm{~s}$.

By contrast, the helium production rate $\mathrm{S}_{\mathrm{He}}$ for the case of $500 \mathrm{MW}$ fusion power is $1.8 \times 10^{20} / \mathrm{s}(0.7 \mathrm{~Pa}-$ $\mathrm{m}^{3} / \mathrm{s}$ ). If the acceptable level of He in the core is $10 \%$, this corresponds to $\sim 1 \times 10^{22} \mathrm{He}$ particles. In case of no helium exhaust, the characteristic time for helium accumulation is $\sim 50 \mathrm{~s}$.

\subsection{Detection limit and accuracy requirements}

ITER specifies an ability to measure the fusion fuel composition (this means the isotopic ratio), as well as the the He (i.e. the fusion $a s h$ ) and impurity concentrations with an accuracy of $20 \%$. Sensitivity is not directly specified. However, based on projected values, this diagnostic system is being designed for expected levels of $\mathrm{He} / \mathrm{D}_{2} \quad(\sim 0.005-0.02$, mainly from the fusion reactions) and $\mathrm{T}_{2} / \mathrm{D}_{2}\left(0.01-10\right.$, but validated with $\mathrm{H}_{2} / \mathrm{D}_{2}$ prototyping) and $\mathrm{Ne} / \mathrm{D}_{2} \quad(\sim 0.01$, if no significant compression).

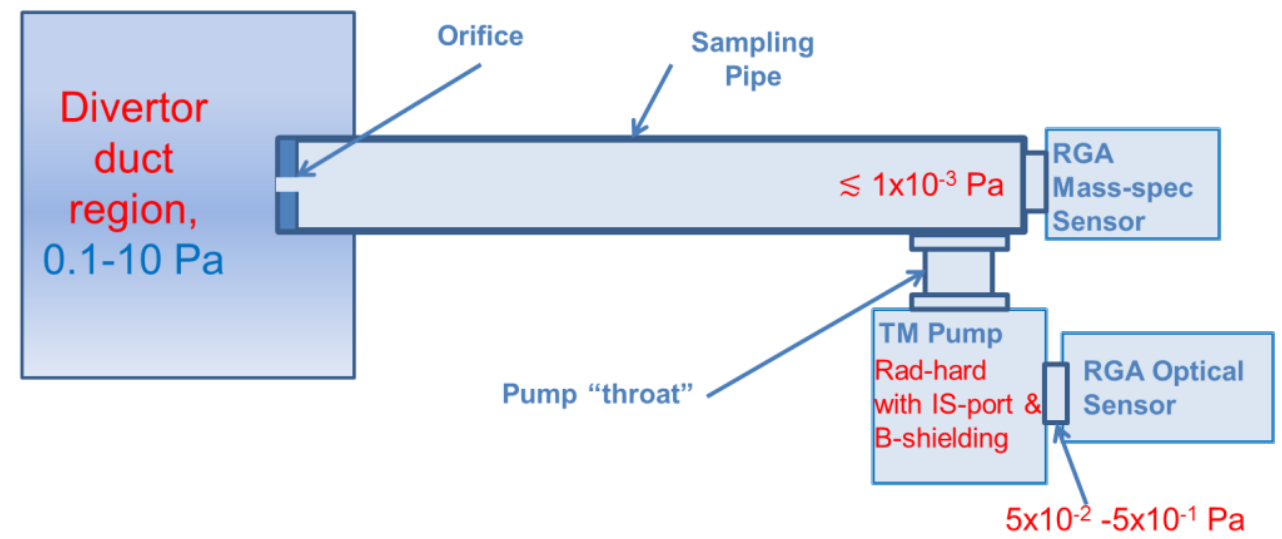

Fig. 1. Functional schematic of the Divertor DRGA.

\section{Divertor DRGA Design}

\subsection{Overview}

Figure 1 is a functional schematic of the Divertor DRGA and serves as the basis of the present design. A critical element of the design is a sampling pipe, whose nominal dimensions of $\mathrm{L}=8 \mathrm{~m}, \mathrm{D}=110 \mathrm{~mm}$ have been determined to provide the best conductance while maintaining the measurement sensors at a serviceaccessible location in the divertor port-cell. Also, two important considerations in reaching this design have been the need to maintain a pressure no higher than $10^{-3}$ $\mathrm{Pa}$ at the location of the mass spectrometer sensors, since operation at higher pressures can significantly limit the sensor lifetimes, and to minimize the flow of the $\left(\mathrm{T}_{2^{-}}\right.$ containing) effluent gas. At the same time, there must be continuous flow to assure measurement during the plasma pulse and also to avoid measurement at a stagnation region. These conditions need to be satisfied for the full range of total pressure values anticipated for the sampled, divertor duct region, which is $0.1-10 \mathrm{~Pa}$.

The present design works by maintaining gas flow in the sampling pipe in the molecular flow regime, with a $\sim 300 \mathrm{~L} / \mathrm{s}$ turbo-molecular (TM) pump providing sufficient pumping speed to maintain the pressure at the sensors in the acceptable range for the mass spectrometer and a $\sim 1$ s response time (based on mass $=4$ for $\mathrm{He}$ or $D_{2}$ ). On the sampling end of the pipe, an orifice is used to develop sufficient pressure drop to achieve the desired pressure on the inlet end of the pipe while maintaining a gas throughput compatible with the design of the sampling pipe and TM pump system.

Maintaining the sampling system in the molecular flow regime limits the flow of effluents to the TM pump, the ITER diagnostics secondary pumping system and the tritium plant. This also allows the above system optimization to be determined analytically with reasonable accuracy. Assuming an ideal orifice (i.e. zero-thickness) the optimized system was based on a 0.25 -mm-dia orifice. Refined calculations, based on real geometry and edge effects, indicate that the same result can be obtained with a manufactured orifice, having D $\sim 0.6 \mathrm{~mm}, \mathrm{~L} \sim 3 \mathrm{~mm}$. In this aspect ratio, one avoids transition regime flow and the conductance is calculated within the short tube approximation.

As discussed in Section 3.4 below, this analytically optimized system design was then modeled numerically using VacTran ${ }^{\circledR}$ [REF 2, VACTRAN] software and shown to meet the response time requirement. 


\subsection{Sampling pipe assembly}

Figure 2 illustrates the physical arrangement of the port cell components of the divertor DRGA. It is noted that the real sampling pipe is comprised of separate pipe sections, which link together other vacuum components, including an isolation valve and a bellows, as well as vacuum seals that provide breakpoints for installation and maintenance. All these components have ITERspecific designs and include double containment with monitored interspaces to prevent tritium leaks into the environment. The detailed design of these components is outside the scope of this paper, as is the engineering analysis used to qualify compatibility with static and dynamic loads. As an extension of the primary vacuum, all sections of the assembly have to be maintained at $200^{\circ} \mathrm{C}$ during bake-out and $100^{\circ} \mathrm{C}$ during plasma operation. Heaters wrapped around external components are not shown in the Figure. The orifice containing section, which traverses the cryostat, is being designed to include its own (vacuum compatible) heater coil.

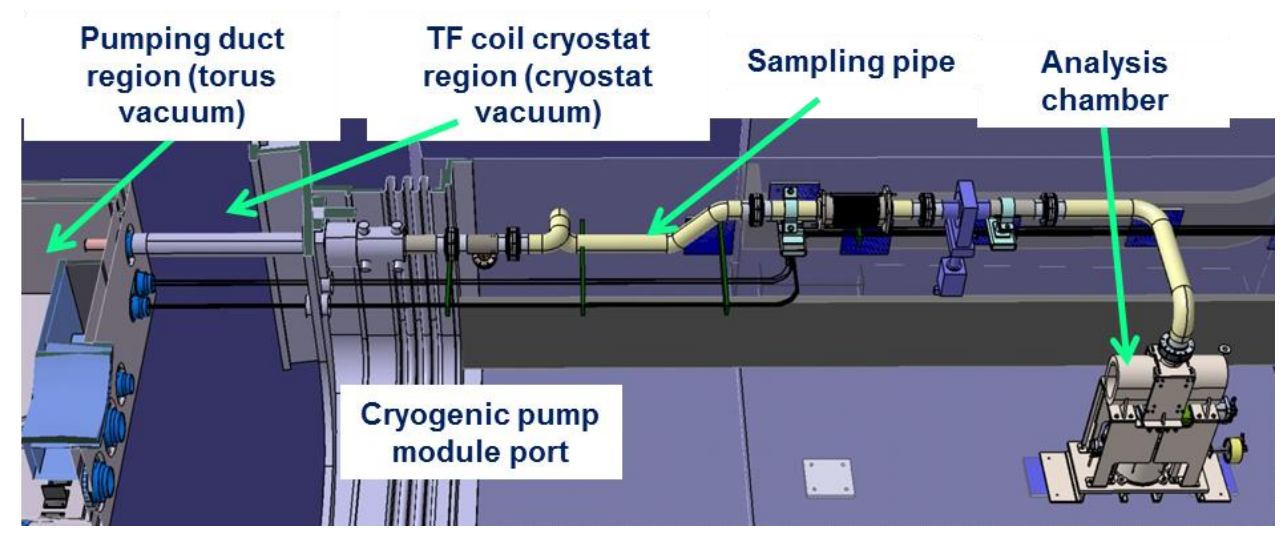

Fig. 2. Illustration of the Divertor DRGA in the environment of the ITER divertor level port cell.

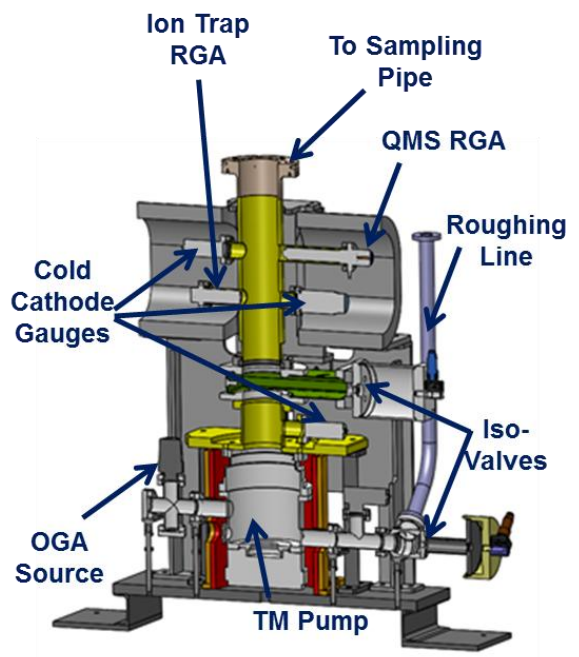

Fig. 3. Cutaway view of the analysis chamber, showing the main cluster of diagnostic sensors, shielded by near-cylindrical, ferromagnetic shielding structures, the TM pump, with the OGA assembly mounted to its inter-stage port and its isolation valves on sensor and roughing sides.

\subsection{Analysis chamber assembly}

Figure 3 illustrates the arrangement of the measurement sensors in an integral part of the differentially pumped chamber, which can be separated from the sampling pipe assembly during installation and maintenance. This analysis chamber assembly includes the TM pump and magnetic shielding structures, with magnetic shielding being the most essential feature of a diagnostics RGA, allowing it to function during a plasma pulse [Ref. 4, KLEPPER '10].

An overview of the three gas analysis techniques that are included in the analysis chamber, as well as a description of a new, laboratory-scale prototype for testing the combined use of these techniques for ITER divertorrelevant measurements, is provided in [Ref. 3, Younkin '04].

The leading choice for the quadrupole mass spectrometer (QMS) RGA at this stage of the design a radiation hardened version of the MKS Microvision 2, specified to resist performance deterioration to $\gamma$ fluences up to $10^{5}$ Gy. This is roughly the fluence anticipated at the sensor for the lifetime of ITER. Most of this will come from nearby divertor water cooling pipes, with activated water flowing during and briefly after the end of each plasma pulse.

A second mass spectrometer is based on the emerging, fully electrostatic, ion trap mass spectroscopy (ITMS) design [Ref. 5, ITMS] The two mass spectrometer will be inside the main shielding structures, on the top section of the chamber, together with cold cathode gauges needed to monitor the operational pressure for the mass spectrometers. This shielding is designed to reduce an up to $0.2 \mathrm{~T}$, mainly vertical, fringing magnetic field, down 
to about $0.005 \mathrm{~T}$, as specified by the manufacturers for unperturbed operation for both types of mass spectrometers.

Unlike the QMS-based mass spectrometer, the recently commercialized (by Granville-Phillips, now part of MKS) ITMS does not have a specified radiation exposure limit. However, it is now possible to operate it with remote electronics, while the compact construction makes it amenable a radiation resistant model by the time of ITER's D-T phase. The ITMS is of particular interest as a complementary (and partly redundant) mass spectrometer, partly due to its capability to scan over a full $100 \mathrm{amu}$ (or even $300 \mathrm{amu}$, full range) in just a fraction of the sampling pipe's response time, allowing for full surveys during the pulse, as well as for its higher sensitivy and resolution at the low masses, where the fusion fuel and ash are located, In fact, early prototype testing has indicated that some discrimination between $\mathrm{He}$ and D2 masses is possible down to $\sim 10 \% \mathrm{He} / \mathrm{D} 2$ concentration, a feature that could be further optimized in collaboration with the manufacturer. This is significant, since the mass difference D2 (4.0271 amu) $\mathrm{He}(4.0026 \mathrm{amu})=0.0245 \mathrm{amu}$ is not resolvable by a standard, full 100 amu QMS sensor. Furthermore, the technology requires calibration with only one calibration gas, greatly reducing cost and complexity.

The third technology integrated into this analysis chamber an optical gas analyzer (OGA). It is presently expected to use the same Penning cold-cathode gauge as the plasma light emission source, as used in past and present implementations to fully discriminate $\mathrm{He}$ from $\mathrm{D}_{2}$, as well as to measure $\mathrm{H}_{2} / \mathrm{D}_{2} / \mathrm{T}_{2}$, using optical spectroscopy of the emission [Refs 6, 7; Klepper ' 97 , Hillis '99], In one implementation, this technology was successfully used on JET in the radiation environment of D-T operation. Unique to the deployment of the OGA in this design is the placement of the OGA source and collection optics at a port between turbine stages of the TM pump. This is done to provide for a higher neutral pressure in the OGA source at an optimal, bright light emission pressure range $\left(5 \times 10^{-2}-5 \times 10^{-1} \mathrm{~Pa}\right)$ while maintaining the mass spectrometers are safe operation pressures $\left(<\sim 10^{-3} \mathrm{~Pa}\right)$ with the differentially pumped chamber arrangement described above.

While shielding the mentioned sensors for magnetic field has been determined to be practical (and mimicking the design of similar sensors operated under similar fringing fields [3]), shielding for the anticipated $n, \gamma$ radiation environment of the port-cell has been deemed impractical (requiring $>\sim 20 \mathrm{~cm}$ of lead shielding, as compared to $<\sim 3-4 \mathrm{~cm}$ for ferromagnetic shielding for the fringing magnetic fields). This is why radiation resistance and remote electronics capability are an important aspect of sensor selection.

Vacuum integrity of the analysis chamber will be monitored with cold cathode gauges. The present design calls for at least one of these gauges to be the radiation tolerant MKS 422 cold cathode gauge. Still under design is a calibration system, intended to be also installed in the port-cell and be operated remotely. It will use known mixtures of gases to calibrate the residual gas analysis and to assure the accuracy of the species concentrations.

\subsection{Numerical simulation of system response}

As mentioned, response time of the system was calculated using conductance, throughput and pressure profiles of the orifice, sampling pipe and TM pump assembly shown in Fig. 2. To reduce uncertainty, the calculation was performed in two ways; using first principle equations solved interactively in MathCad and with the widely used vacuum simulation code VacTran ${ }^{\circledR}$. Both approaches yield response times on the order of $1 \mathrm{~s}$. Figure 4 shows the most relevant output of the VacTran ${ }^{\circledR}$ code, clearly showing that the pressure is, in fact, maintained low enough at the sensor cluster for the RGA sensors and that the response time remains $\sim 1 \mathrm{~s}$ for the full range of envisioned pressures in the sampled region of the pumping duct.

It is worth noting that the response times discussed above are both based on macroscopic models of gas flow in the pipework. Gas-surface interactions are not included in such calculations and would be difficult to simulate. For this reason, the near-term R\&D plan includes fabrication of a full scale mock-up of the sampling pipe described above. Tests, in combination with the recently assembled DRGA prototype, will build confidence in the models or, failing that, allow the models to be better correlated with numerical analysis.

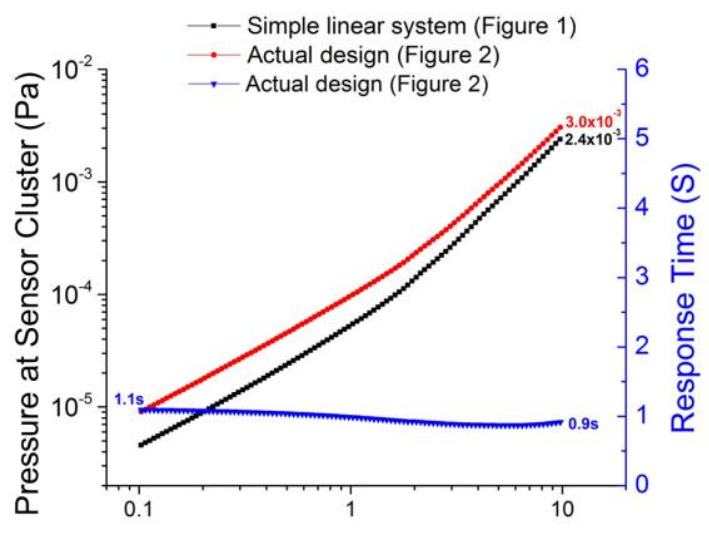

Pressure in Divertor Pumping Duct $(\mathrm{Pa})$

Fig. 4. VacTran ${ }^{\circledR}$ numerical simulation of pressure at the sensor cluster and corresponding response time, as a function of the pressure in the sampled region (the divertor pumping duct). The pressure is simulated for both a simple, linear pipe as shown in the schematic of Fig. 1 (black data) and for one including the elbow and manifold sections of the actual system, shown in Fig. 2 (red data), to get an idea of the effect of actual geometry. In both cases, a real (physical) orifice is included ( $\sim 3 \mathrm{~mm}$ length with $0.6 \mathrm{~mm}$ DIA.). 


\section{Summary, plans forward and $R \& D$ needs}

It is seen that design of the divertor DRGA system is approaching a mature stage in this early part of the Final Design phase of the project. An innovative arrangement for the differentially pumped analysis chamber, which maintains the main chamber at mass-spectrometer range of pressure and then raises back the pressure for the OGA using an inter-stage port on the TM pump, allows for meeting the response time requirement with a single, reasonably sized pump. Not discussed here is compatibility of the choice and arrangement of sampling pipe components with all static and dynamic loads representative of the location of the system in the divertor port-cell. A full analysis of the response of the system (up to the analysis chamber) was carried out as part of the recent ITER DRGA Divertor Sampling Pipe Final Review (July 29th \& 30th, 2014, at ITER Organization headquarters). Similar analysis, but including the analysis chamber assembly, is planned for a separate review in Sep-2015.

\subsection{R\&D activities, past and near future}

In addition to the mentioned experimental validation of the response time, anticipated near-term R\&D activities include sensitivity testing (compatible with the values given in Section 2.2), development of efficient calibration techniques, compatible with ITER operations, and experimental validation of IS port operation of the OGA.

\section{Acknowledgments}

Continuing efforts by S. Vartanian, CEA-IRFM, France and U. Kruezi, CCFE, UK, to encourage and support testing of innovative technologies related to this ITER project on Tore Supra/WEST and JET, correspondingly, are greatly appreciated.

\section{References}

[1] A.J.H. Donne et al, "Progress in ITER Physics Basis, Ch7: Diagnostics, in special issue PROGRESS IN THE ITER PHYSICS BASIS, Nucl. Fus. 47(6) 2007, p337$384 "$.

[2] VacTran Version 3.64.2, Professional Engineering Computations, Livermore, CA; http://www.vactran.com/

[3] C. C. Klepper, D. L. Hillis, J. Bucalossi, D. Douai, P. Oddon, and S. Vartanian, Rev. Sci. Instrum. 81, 10E104 (2010). http://dx.doi.org/10.1063/1.3474660.

[4] T. R. Younkin, T. M. Biewer, C. C. Klepper and C. Marcus, Rev. Sci. Instrum. 85, $11 \mathrm{E} 816 \quad$ (2014); http://dx.doi.org/10.1063/1.4892157.

[5] A. V. Ermakov and B. J. Hinch, Rev. Sci. Instrum. 81, 013107 (2010). http://dx.doi.org/10.1063/1.3276686

[6] C. C. Klepper, D. L. Hillis, M. R. Wade, R. Maingi and G. R. McKee, Rev. Sci. Instrum. 68, 400 (1997); http://dx.doi.org/10.1063/1.1147839

[7] D. L. Hillis, P. D. Morgan, J. K. Ehrenberg, M. Groth, V. Kumar, M. F. Stamp, and M. von Hellerman, Rev. Sci. Instrum. $\quad \mathbf{7 0}(1), \quad 359-362 \quad$ (1999). http://dx.doi.org/10.1063/1.1149301 